\title{
Physical Map of the Human Genome
}

National Cancer Institute

\section{Source}

National Cancer Institute. Physical Map of the Human Genome. NCI Thesaurus. Code C16056.

A map of the locations of identifiable landmarks on DNA (for example, restriction enzyme cutting site, genes), regardless of inheritance. Distance is measured in base pairs. For the human genome, the lowest-resolution physical map is the banding patterns on the 24 different chromosomes, the highest-resolution map would be the complete nucleotide sequence of the chromosomes. 